\title{
Good Evolution of Stage IV Neuroblastoma in the Absence of Stem Cell Autograft
}

\author{
Wala Ben Kridis ${ }^{1 *}$, Ines Werda ${ }^{1}$, Rim Kallel ${ }^{2}$, Nabil Toumi ${ }^{1}$, Tahia Boudawara ${ }^{2}$, Afef Khanfir ${ }^{1}$ and Mounir Frikha ${ }^{1}$ \\ ${ }^{1}$ Department of Oncology, Habib Bourguiba Hospital, Tunisia \\ ${ }^{2}$ Department of pathology, Habib Bourguiba Hospital, Tunisia
}

Received: 阱 May 29, 2018; Published: 阱June 11, 2018

*Corresponding author: Wala Ben Kridis, Department of oncology, Habib Bourguiba Hospital, Sfax Tunisia

\begin{abstract}
Neuroblastoma is a relatively common pediatric pathology of the sympathetic nervous system. The particularity of neuroblastoma lies in its development from cells whose embryological maturation into adult sympathetic nerve cells or chromaffin cells is incomplete. In the literature, many cases of spontaneous regression of neuroblastoma have been reported, but the mechanisms for which they are responsible remain ambiguous. The specificity of our case is the benign evolution of an osteomedullary stage 4 neuroblastoma and its maturation into a ganglioneuroma 6 years after the end of chemotherapy. This transformation depends on various factors related to the tumor (such as the over expression or not of N-myc, the presence or absence of Treks and their receptors), the host (the intervention of the immune system) and to other external factors. Being able to induce this differentiation by not leaving it to chance would be a therapeutic challage and could categorically change the prognosis of neuroblastomas. This merits a lot of research and studies of various potential future treatments (such as treks inhibitors and retinoid).
\end{abstract}

Keywords: Neuroblastoma; Ganglioneuroma; Etiopathogeny.

Abbreviations: NGF: Nerve Growth Factor; BDNF: Brain Derived Neutrophic Factor; VMA: Vanyl Mandelic Acid; NB: Neuroblastoma.

\section{Introduction}

Neuroblastoma (NB) is a relatively common pediatric pathology of the sympathetic nervous system [1]. It is a malignant tumor of the neuroblast.

The originality of neuroblastoma lies in its development from cells whose embryological maturation into adult sympathetic nerve cells or chromaffin cells is incomplete. In addition, the evolution of neuroblastoma is unlikely: the tumor can be aggressive and evolves rapidly into a metastatic disease, as it can be "nice" and undergoes regression or maturation (spontaneous or probably induced) into a benign tumor: the ganglioneuroma. Spontaneous regression of neuroblastoma is rare, occurring in 1 to $2 \%$ of cases. However, this tumor has the highest rate of spontaneous regression than any other cancer [2]. The incidence of spontaneous regression in neuroblastoma is estimated to be between 10 and 100 times greater than that of any other human cancer [3]. In the literature, many cases of spontaneous regression of neuroblastoma have been reported, but the mechanisms for which they are responsible remain ambiguous. Several theories have been advanced in order to solve this enigma, and to try to find some therapeutic approaches. We report the case of a female patient aged 21 months at the time of diagnosis, who was followed and treated for stage 4-neuroblastoma. The evolution, after six years of stopping treatment, was marked by the maturation of its tumor into a ganglioneuroma. From this case, we want to identify the various mechanisms that could explain this phenomenon which would encourage pushing the studies in this direction and try to induce the maturation or the regression of the tumor really and not to wait for its spontaneous occurrence desperately during the evolution of the disease.

\section{Case Report}

A 21-month-old patient was admitted in October 2006 for exploration of a prolonged fever at $40^{\circ}$. The clinical examination was normal. In biology she had norm chromic normocytic anemia at $6.9 \mathrm{~g} / \mathrm{dl}$ with thrombocytopenia at 10,000 . The sternal puncture showed a very poor marrow with the presence of rarely isolated malignant cells often grouped in rosettes evoking an invasion by a neuroblastoma. Urinary vanyl mandelic acid (VMA) was increased 
to $27 \mathrm{mg} / 24$ hours. The thoracoabdominopelvic CT scan showed a large mass measuring $7.5 * 4.2 \mathrm{~cm}$ at the left adrenal compartment (Figure1). Bone scintigraphy showed hyper fixation of the left parietal bone and the right tibia. Meta-iodobenzylguanidine (MIBG) scintigraphy revealed fixation of the superior pole of the left kidney and the left parietal bone. The osteomedullary biospie concluded to neuroblastoma. The diagnosis was stage 4 neuroblastoma. N-myc was negative.

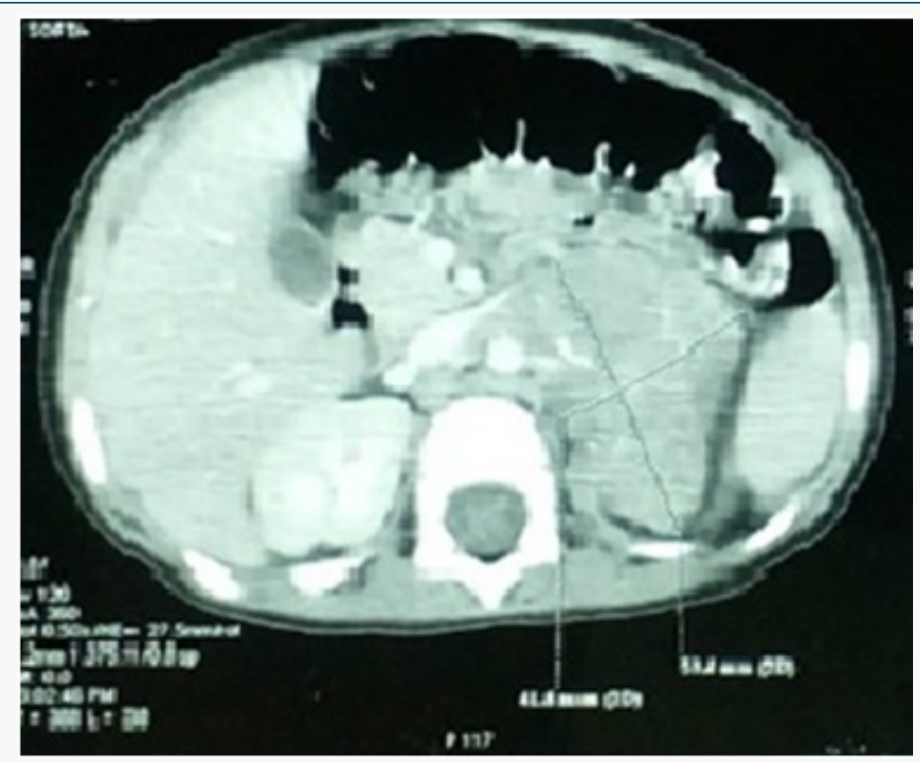

Figure 1: Abdominal CT.

Voluminous hypo dense mass of the left adrenal lining of $75 * 42 * 39 \mathrm{~mm}$ with fine peripheral calcifications, pushing back the left kidney, the spleen and the pancreas; encompassing the renal pedicle, evoking a neuroblastoma.

The patient had 6 courses of alternating chemotherapy Etoposide-ciplatin and CADO (cyclophosphamide, dactinomycin, doxorubicin and vincristine) completed in March 2007 with negotiation of urinary VMA. CT showed a tumor residue of $5 \mathrm{~cm}$ with 2 secondary lung nodules with a stable appearance of the
MIBG scintigraphy. The decision was to stop chemotherapy. The patient was followed regularly at our consultation. Residual mass surgery was proposed, but was considered difficult and risky by the surgeons.

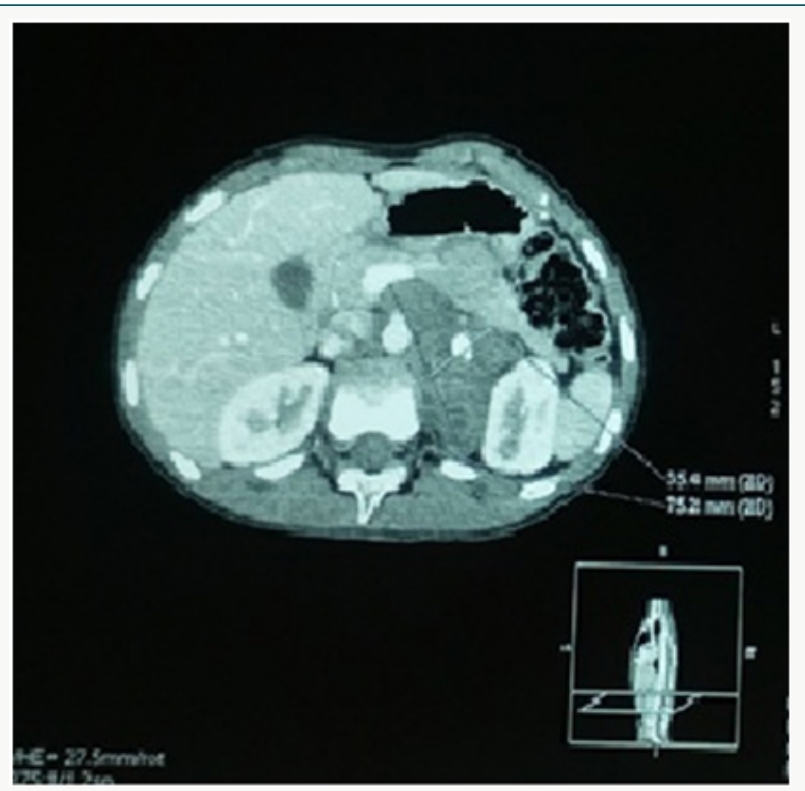

Figure 2: Abdominal CT scan.

Left adrenal mass measuring $75^{*} 35 \mathrm{~mm}$ in an axial plane and $65 \mathrm{~mm}$ in a coronal plane, which is seat of a central macrocalcification and some micro-calcifications ; and which engages the aorta, the celiac trunk, the superior mesenteric artery, the left renal artery and vein. 


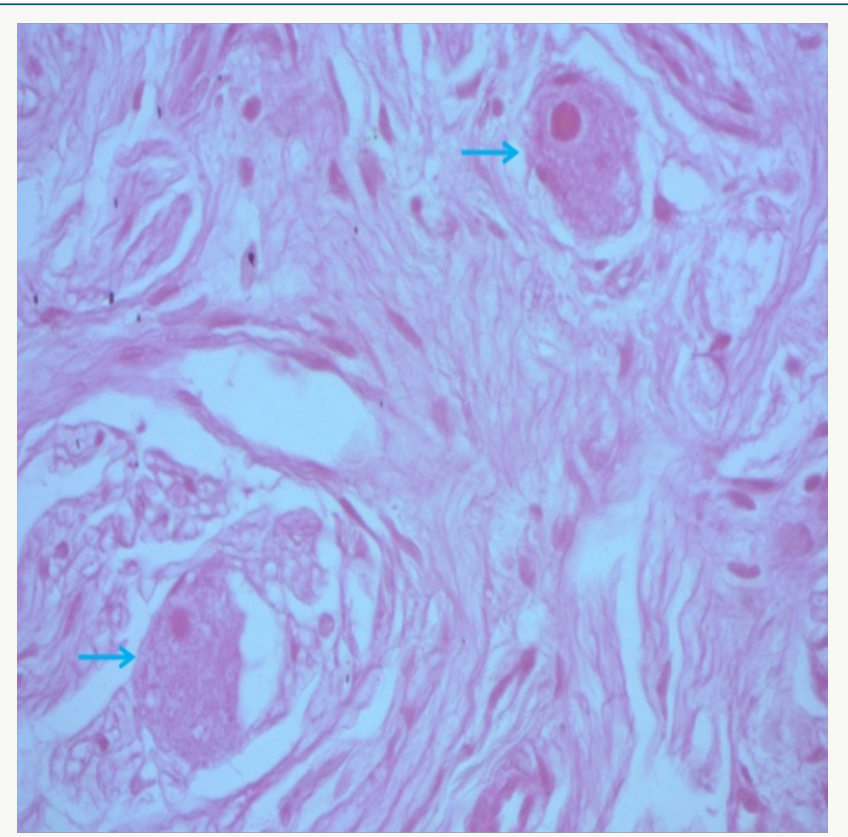

Figure 3: Proliferation of fusiform cells interspersed with large cells with dark ctoplasm and large nuclei (Blue arrow: ganglion cells) (Hex 400).

The evolution was marked by an increase in the size of the left retro peritoneal mass on the CT scan done in December 2013 (Figure 2), with disappearance of the left adrenal fixation and persistence of left parietal fixation on MIBG scintigraphy. Anatomopathologic study of a left adrenal biopsy under CT scan found a ganglioneuroma (Figure 3). Currently, our patient is 12 years old, in good health, with good growth and normal schooling.

\section{Discussion}

Neuroblastoma and ganglioneuroma are two tumors derived from nerve embryonic cells being maturation. They differ in their degree of differentiation and prognosis. Ganglioneuroma is a benign tumor that is composed of gangliocytes and mature stroma. Neuroblastoma is a malignant, immature, undifferentiated and aggressive tumor. Despite its aggressiveness, this tumor may have a benign later evolution, even in case of metastatic disease, undergoing involution or maturation into a benign tumor. Until now, the mechanisms of regression or maturation of neuroblastoma remain poorly defined.

From our reported case, we will try to analyze the different mechanisms proposed in the literature that could explain the phenomenon of differentiation of neuroblastoma into a ganglioneurema as it happened with our patient.

The growth and differentiation of neuroblastoma cells is regulated by certain genes and vital proteins. N-Myc is a protooncogene that is over-expressed in some human cancers. When subjected to mutations or over-expression, it stimulates cell proliferation and behaves like an oncogene.

Over expression of $\mathrm{N}$-myc represents an unfavorable progression factor. The amplification of $\mathrm{N}$-myc is detected in
16 to $25 \%$ of neureoblastomas [4]. High levels of N-myc are associated with accelerated tumor proliferation and metastatic neuroblastoma behavior [5]. Unlike its absence promotes apoptosis, decreases tumor proliferation and stimulates terminal neuronal differentiation. In our case, N-myc wasn't over expressed, which would be a booster element to the maturation.

Tropomyosin-related kinase: $\operatorname{TrK} \mathrm{A}$ and $\operatorname{TrK} \mathrm{B}$ are tyrosine kinase receptors, whose legends are: the nerve growth factor (NGF) and the brain derived neutrophic factor (BDNF) respectively [6]. These receptors have critical roles in the development and maintenance of the central and peripheral nervous system. They also have an important role in neuroblastome pathogenesis. In the early 1990s, Trk-receptors were identified as important prognostic factors influencing the heterogeneous clinical behavior of NB $[7,8]$. Morphologic differenciation of neuroblastoma may involve the interaction between NGF and its receptors [8].

Studies have shown that high TrK A expression is associated with favorable clinical and laboratory characteristics (young age, lower stage and negative $\mathrm{N}$-myc). Its expression thus represents a factor of good prognosis and is linked to a better survival [7]. While $\operatorname{TrK} \mathrm{B}$, highly expressed, is a pejorative factor, and would be associated with aggressive neuroblastomas with amplified N-myc. In addition, studies have shown that the positivity of $\operatorname{TrK} A$ in neuroblastoma cells induces a slowing of proliferation [8,9] and promotes differentiation in the presence of its legend: NGF [7]. On the other hand, TrK B induces the opposite effect and also gives rise to resistance to chemotherapy [7].

Thus, TrK A signaling and or lack of TrK B signaling would be a promoter factor for neuroblastoma maturation. According to these reflections, being able to act on these receptors or their 
legends could influence the natural evolution of neuroblastoma, and induce a welcome regression or maturation of the tumor. A profound understanding of TRK signaling is advisable before using Trks therapeutic targets in neuroblastoma. Currently, several TrK inhibitors are in Phase 1 clinical trials. In our institute, we don't test TrK. Retinoid are also an alternative that should be mentioned because of its tested and proven ability in vitro to induce differentiation and decrease proliferation in neuroblastoma cells [10]. Moreover, retinoid isotretinoin has been incorporated in the treatment of high-risk neuroblastoma in order to induce neuroblastoma cell maturation. The mechanism of action is not clear, but it seems that these retinoid increases the expression of TrK receptors. Our patient did not receive retinoid, so we can exclude their involvement in maturation. It would also be important to think about the behavior of the immune system towards neuroblastoma cells. It seems that this system is able to specifically recognize an epitope secreted by neuroblastic cells, which would induce their clearance and elimination by the cells of immunity. More detailed studies are needed to better define this epitope which could present a possible therapeutic target. Also, it would be interesting to examine the role of other host factors such as polymorphism in human leukocyte antigen [11].

\section{Conclusion}

The specificity of our case is the benign evolution of an osteomedullary stage 4-neuroblastoma and its maturation into a ganglioneuroma 6 years after the end of chemotherapy. This transformation depends on various factors related to the tumor (such as the over expression or not of $\mathrm{N}$-myc, the presence or absence of Trks and their receptors), the host (the intervention of the immune system) and to other external factors. Being able to induce this differentiation by not leaving it to chance would be a therapeutic challage and could categorically change the prognosis of neuroblastomas. This merits a lot of research and studies of various potential future treatments (such as trks inhibitors and retinoid).

\section{References}

1. Zhi-Gang Yao, Xiu-Mei Liu, Ye-Jun Qin (2016) Spontaneous Maturation of Neuroblastoma to Ganglioneuroma: Two Case Studies. Fetal and Pediatric Pathology 35(6).

2. Prichard J, Hickman JA (1994) Why does stage 4s neuroblastoma regress spontaneously? Lancet 344(8926): 869-870.

3. Sitarz AL, Santulli TV, Wigger HJ, Berdon WE (1975) Complete maturation of neuroblastoma with bone metastases in documented stages. J Pediatr Surg 10(4): 533-536.

4. Westermark UK, Wilhelm M, Frenzel A, Henriksson MA (2011) The MYCN oncogene and differentiation in neuroblastoma. Semin Cancer Biol 21(4): 256-266.

5. Huang M, Weiss WA (2013) Neuroblastoma and MYCN. Cold Spring Harb Perspect Med 3(10): a014415.

6. Light JE, Koyama H, Minturn JE, Ho R, Simpson AM, et al. (2012) Clinical significance of NTRK family gene expression in neuroblastomas. Pediatr Blood Cancer 59(2): 226-232.

7. Schramm A, Schulte JH, Astrahantseff K, Apostolov O, Limpt Vv, et al. (2005) Biological effects of TrkA and TrkB receptor signaling in neuroblastoma. Cancer Lett 228(1-2):143-153.

8. A Nakagawara, Arima MN, Scavarda NJ, Azar CG, Cantor AB (1993) Association between high levels of expression of the Trk gene and favorable outcome in human neuroblastomas. N Engl J Med 328(12): 847-854.

9. Kim CJ, Matsuo T, Lee KH, Thiele CJ (1999) Up-regulation of insulin-like growth factor-II expression is a feature of TrkA but not TrkB activation in SH-SY5Y neuroblastoma cells. Am J Pathol 155(5): 1661-1670.

10. Matthay KK, Villablanca JG, Seeger RC, Stram DO, Harris RE, et al. (1999) Treatment of high-risk neuroblastoma with intensive chemotherapy, radiotherapy, autologous bone marrow transplantation, and 13-cisretinoic acid. Children's Cancer Group. N Engl J Med 341(16): 11651173.

11. Cheung, NK V, Dyer MA (2013) Neuroblastoma: Developmental biology, cancer genomics and immunotherapy. Nat Rev Cancer 13(6): 397-411.
This work is licensed under Creative Commons Attribution 4.0 License

To Submit Your Article Click Here:

Submit Article

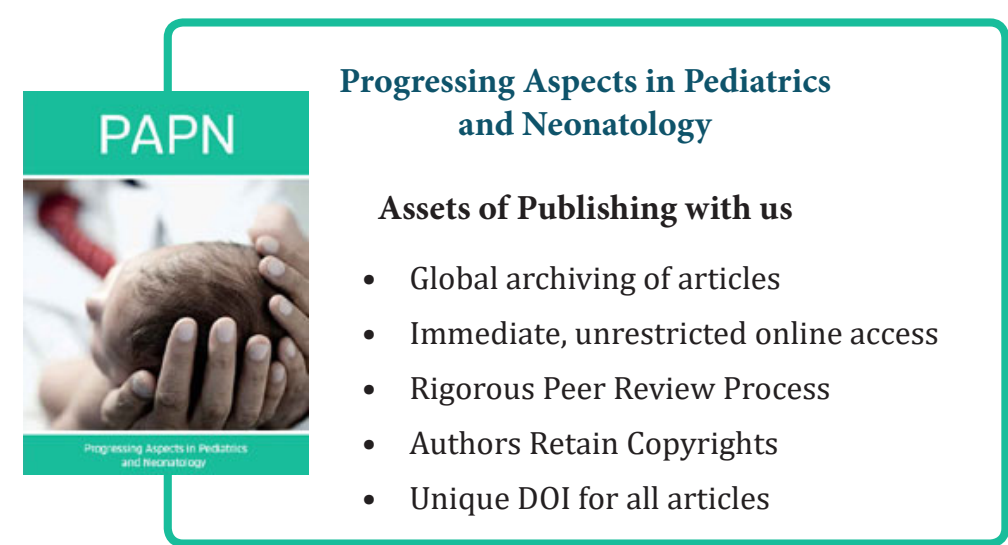

